\title{
Finding the right lenses
}

Over the past several decades, classical molecular biology and cytogenetic techniques have enabled the study of DNA sequence variation at the smallest and largest scales of size. Between these two extremes, and beyond the reach of classical approaches, lay, until very recently, a largely uncharted realm involving variation of intermediate scale. Advances in technology have now begun to reveal this treasure-trove of large-scale, submicroscopic variation, and the impact on the field of human genetics has been dynamic and far-reaching.

$\mathrm{F}$ rom the earliest cytogenetic studies, it was recognized that losses or gains of entire chromosomes or microscopically visible alterations involving very large chromosomal segments were the cause of several common developmental disorders, including Down's syndrome. Beginning in the early 1990s, it also became apparent that submicroscopic gains or losses of specific chromosomal segments were a recurrent cause of many other rare genetic diseases. What was generally unappreciated, up until a few years ago, was the extent to which focal variation in DNA copy number contributed to the range of normal sequence variation among apparently healthy individuals.

In 2004, two landmark studies, by Sebat et al. (Science 305, 525-528; 2004) and Iafrate et al. (Nat. Genet. 36, 949-951; 2004), brought the landscape of large-scale copy-number variation in the human genome into clear focus, revealing a range of genetic diversity that has since sparked intense efforts to characterize the extent of such variation in the human population and to understand what role it has in phenotypic variation and disease susceptibility. In parallel, driven by many of the same technologies, there has been a strong resurgence in studies in examining the role of de novo large-scale structural changes in sporadic disease.

In this issue, we present a collection of eight commissioned pieces highlighting recent advances in the field of human genomic structural variation. In an opening Commentary, Jonathan Sebat considers how the discoveries of the last few years have altered the landscape of genetic research, and what impact this will have on the field going forward. In the first of seven Perspectives, Stephen Scherer and colleagues discuss challenges in integrating data from existing surveys of structural variation, and propose standards and guidelines to facilitate ongoing efforts to catalog the range of structural variation in the human population and to unravel the phenotypic consequences of this variation. Nigel Carter follows with a discussion of existing platforms and methods for analyzing copy-number variation using microarrays, offering glimpses of the emerging array-based and sequencing platforms that will soon yield increasingly high resolution views of variation across all scales. Evan Eichler and colleagues analyze the distribution and genomic properties of known structural variants and consider the role of mutational and selective effects in driving the population and evolutionary dynamics of structural variation. Following on a similar theme, Donald Conrad and Matthew Hurles examine current studies of structural variation from a population perspective, noting biases in the ascertainment of structural variants and the limited sampling, so far, from diverse human populations, while emphasizing the value of developing a population genetics framework for understanding the role of structural variants in adaptation and phenotypic variation.

In the first of the three Perspectives focused on disease, Steven McCarroll and David Altshuler discuss challenges in incorporating analyses of copy-number variants into association studies of human disease, noting the difficulty of defining precise allelic states while also highlighting the statistical challenges in assessing the contribution of rare copy-number variants to disease phenotypes. Shifting focus from common copy-number polymorphisms to de novo rearrangements, Jim Lupski argues from existing data that locus-specific mutation rates for genomic rearrangements may be orders of magnitude greater than nucleotide-specific rates for base substitutions, underscoring the potentially widespread contribution of such rearrangements to sporadic disease. Finally, writing from the perspective of clinical cytogeneticists, Charles Lee and colleagues offer practical advice on how to apply studies of copynumber variation in the area of clinical diagnostics.

We thank the authors for contributing these thoughtful and insightful pieces, and our reviewers for their helpful feedback. We also thank our sponsor NimbleGen for helping us to produce this supplement and make it available to readers in print and online. To our readers, we hope you enjoy this fresh glimpse into the exciting realm of human genomic structural variation. 